\title{
High Intensity Regimes for Resonant Raman Compression
}

\author{
N. J. Fisch ${ }^{a}$, V. M. Malkin ${ }^{a}$, and Z. Toroker ${ }^{b}$ \\ ${ }^{a}$ Department of Astrophysical Sciences, Princeton University, Princeton, NJ USA 08540; \\ ${ }^{b}$ Department of Electrical Engineering, Technion Israel Institute of Technology, Haifa 32000, \\ Israel
}

\begin{abstract}
In order to achieve the largest laser intensities, a plasma might be used as the amplification medium, thereby avoiding the material limits of conventional materials. The technique considered is resonant backward Raman amplification in plasma, wherein a short counter-propagating seed pulse, with frequency downshifted from a long pump pulse by the plasma frequency, absorbs the pump energy through a resonant decay interaction of the two counter-propagating light waves and a plasma wave. In the pump-depletion regime, the counter-propagating seed pulse assumes a self-contracting self-similar form, capturing the pump energy in a pulse of far shorter duration. This technique encounters limitations both at high laser seed output intensities and high pump laser intensities. At high seed output intensities, there are modulation instabilities that break up the output seed. At high pump intensities, the resonant interaction is interrupted by wavebreaking of the plasma wave. These limitations, while limiting, may not be as limiting as might be at first thought.
\end{abstract}

\section{INTRODUCTION}

The technique of chirped pulse amplification (CPA) achieves the largest laser intensities to date. ${ }^{1}$ This technique, however, is subject to the material limits of conventional materials. In the CPA technique, a laser pulse is stretched, then amplified, and then finally re-compressed. This technique has been recently reviewed. ${ }^{2}$ At very high laser intensity and fluence, conventional optical materials fail, limiting the intensity of the output pulses. For wavelengths on the order of a micron and pulse lengths on the order of picoseconds, the maximum intensity is limited to tens of $\mathrm{TW} / \mathrm{cm}^{2}$. This limit is incurred by the material properties of the gratings employed to stretch and re-compress the pulse. ${ }^{3}$ For shorter wavelengths, this technique becomes much more difficult altogether for high intensity applications because the material gratings become even more fragile.

These material limits may be overcome through the use of plasma as a medium mediating resonant backward Raman amplification in plasma. In this technique, as depicted in Fig. 1, a short counter-propagating seed pulse, with frequency downshifted from a long pump pulse by the plasma frequency, absorbs the pump energy through a resonant decay interaction of the two counter-propagating light waves and a plasma wave. In the pumpdepletion regime, the counter-propagating seed pulse assumes a self-contracting self-similar form, capturing the pump energy in a pulse of far shorter duration. ${ }^{4}$ Absent non-ideal effects, the so-called $\pi$-pulse regime is attained, wherein the amplified seed pulse comprises a series of spikes, with most of the energy contained in the leading spike. Robust amplification, avoiding deleterious effects such as premature backscatter from noise $\mathrm{e}^{5-8}$ or precursors ${ }^{9}$ to the amplified pulse, requires careful selection of plasma and wave parameters, ${ }^{10}$ including techniques of chirping the seed and pump pulses. ${ }^{11}$

There are, however, limiting effects that enter at both high seed output intensities and at high pump intensities. The primary objective of this paper is to review recent work on this topic. ${ }^{12-14}$

At high seed output intensities, relativistic effects tend to break up the pulse, particularly the largest, leading spike, limiting the compression effect. ${ }^{12}$ The best course would then appear to be to terminate the plasma, since a longer plasma would then only lead to a much less desirable output seed pulse, less intense, broken up, and

NJF: E-mail: fisch@princeton.edu

VMM: E-mail: malkin@astro.princeton.edu

ZT: E-mail: ztoroker@tx.technion.ac.il

Relativistic Plasma Waves and Particle Beams as Coherent and Incoherent Radiation Sources

edited by Dino A. Jaroszynski, Proc. of SPIE Vol. 9509, 95090G · C 2015 SPIE

CCC code: $0277-786 X / 15 / \$ 18 \cdot$ doi: $10.1117 / 12.2183062$

Proc. of SPIE Vol. 9509 95090G-1 


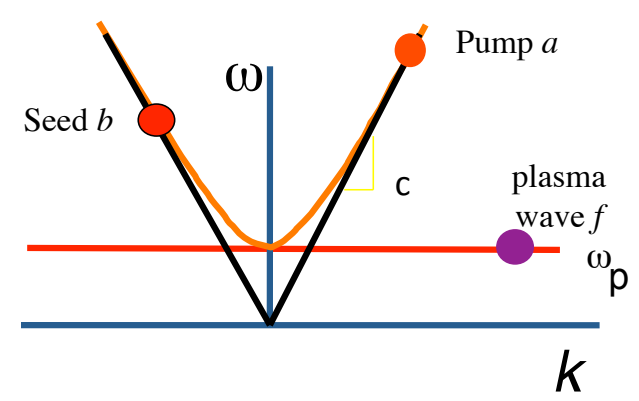

Figure 1. The counterpropagating geometry in $\omega-k$ space for resonant Raman compression. The pump $a$, the seed $b$, and the Langmuir wave $f$ are in resonance. For large ratios of pump to plasma frequencies, the plasma wave wavenumber is approximately twice the pump laser wavenumber.

less focusable. However, it turns out that after the break-up of the leading spike, subsequent spikes can in fact reach higher intensity yet. ${ }^{13}$ This suggests that a longer amplification length may then be advantageous after all. However, the plasma conditions and laser parameters need to be carefully prepared to realize this regime.

At high pump intensities, which can be useful in achieving the compression effect with less plasma, the plasma wave can be broken, limiting the efficiency. However, even at moderate wavebreaking, the compression can still be efficient, only becoming inefficient in the deep wavebreaking regime. ${ }^{14}$ In fact, moderately efficient Raman amplification can be obtained for pump intensity beyond the wavebreaking threshold, in fact up to about four times the wavebreaking threshold. This regime might be called the weak wavebreaking regime. The temperature, of course, must also be low enough to avoid Landau damping. However, for pump intensity larger than four times the wavebreaking threshold, the efficiency of the amplification is significantly decreased. This regime might be called the strong wavebreaking regime. It also turns out ${ }^{14}$ that, in the weak wavebreaking regime, the Raman amplification efficiency can be improved by utilizing a more intense initial seed pulse. In the strong wavebreaking regime, however, a strong initial seed pulse will not strongly affect the Raman amplification efficiency. Findings in the archival literature are consistent in the weak wavebreaking regime. In the strong wavebreaking regime, however, there still appears to be some variance within the archival literature on the achievable efficiency.

This paper is organized as follows: In Sec. 2, we review the historical background of Raman compression effect and related effects in plasma for compression of high intensity pulses. In Sec. 3, we write the model equations and discuss numerical approaches. In Sec. 4, we describe the regime below wavebreaking, where the relativistic nonlinearity plays a role. In particular, numerical examples of the extended regime are given, showing how the secondary spikes dominate. In Sec. 5, we consider the wavebreaking regime. In Sec. 6, we summarize the major results.

\section{HISTORICAL BACKGROUND}

The method of resonant Raman backscattering ${ }^{4}$ permits, in theory, intensities far greater than can be obtained by CPA, since plasma can tolerate intensities $10^{5}$ higher than material gratings before deleterious effects, associated with the relativistic motion of electrons, set in. It also, in theory, can be highly efficient, since complete pump depletion should be achievable, with only a fraction of the pump energy left in the plasma wave. That fraction, set by the Manley-Rowe relation, is the ratio of plasma frequency to pump frequency. Other plasma-based mechanisms have now also been proposed to compress lasers in a counter propagating geometry, including the use of Compton scattering ${ }^{15}$ or, more recently, the use of a strong-coupling backward Brilliouin interaction ${ }^{16,17}$ or possibly a mixture of Raman and Brilliouin effects. ${ }^{18}$ However, at present, the resonant Raman effect ${ }^{4}$ has enjoyed the most theoretical and experimental development, and appears to be the most promising for high intensity applications.

Since the Raman amplification requires the plasma wave to mediate the energy transfer, the transfer can be destroyed by processes that interfere with the resonance or that extinguish the plasma wave. Thus, both Landau damping and Langmuir wavebreaking ${ }^{19}$ can reduce significantly the Raman amplification efficiency. 
Landau damping occurs when the phase velocity of the Langmuir wave is on the same order of the electron thermal velocity. Langmuir wavebreaking occurs when the longitudinal quiver electron velocity exceeds the phase velocity of the Langmuir wave. In both processes, the Langmuir wave transfers part or most of its energy to the electrons, and is then unavailable to mediate the resonant energy transfer. The breaking of the Langmuir wave occurs more readily at low plasma density, and it depends directly on the intensity of the plasma wave; the intensity of the plasma wave in turn is proportional (by the ratio of their frequencies) to the pump intensity in the pump depletion regime. Thus, experimental and theoretical attention has been paid mostly for pump intensities low enough to avoid the wavebreaking.

In the regimes that avoid wavebreaking, the backward Raman efficiency is impeded by deleterious effects such as seed dispersion, electron relativistic nonlinearity, and plasma homogeneity, for which theoretical remedies can be proposed as well. ${ }^{11,20-24}$ The key plasma parameters for this favorable regime, including the parameters that produce remedies for the identified deleterious effects, were recently summarized. ${ }^{10}$ In accordance with the theoretical expectations, the Raman amplification effect was demonstrated most successfully in regimes that avoid wavebreaking. ${ }^{25-32}$ To achieve the maximum possible amplification efficiency, the major constraint respected in these experiments was that the pump intensity was below the wavebreaking threshold. When this constraint was not respected, the efficiency clearly decreased. ${ }^{30,33,34}$

Nonetheless, apart from the issue of wavebreaking, there would be clear advantages to operating in the parameter regime prone to wavebreaking. At large laser to plasma frequency ratio, the Langmuir phase velocity is small, making wavebreaking or Landau damping more likely, but such a ratio is useful in that Raman forward scattering is lessened. Also, there are advantages too in larger pump intensities, since the amplified seed pulse grows faster, so that less plasma need be used to mediate the interaction. But the combination of these advantages does make wavebreaking more likely. Thus, it also becomes important to see how large an efficiency can in fact be attained in this regime. There are promising simulations in this regime, ${ }^{35}$ although other simulations, in the deep wavebreaking regime, report smaller efficiencies. ${ }^{14}$

The above considerations all apply to the optical wavelengths of about 1 micron. However, a further incentive to study the wavebreaking regime is that this regime is invariably reached for very short wavelength applications such as Raman compression of UV and X-ray lasers. ${ }^{36}$ In those regimes, it is hard to construct a medium without a large laser to plasma frequency ratio, so it becomes particularly challenging to respect the wavebreaking constraint.

\section{MODEL DESCRIPTION}

Absent wavebreaking, the major physical effects associated with the resonant Raman compression regime can be captured by the 3 -wave coupled envelope equations. Using the fact that there are many laser wavelengths in the pump pulse and at least several laser wavelengths in the short seed pulse, the solution to these equations may be envisioned as the interaction between slowly-varying wave envelopes. The resulting one-dimensional equations for the resonant 3-wave interaction, taking into account the lowest order relativistic electron nonlinearity and group velocity dispersion effects for the amplified pulse, can then be put in the form: ${ }^{37}$

$$
\begin{gathered}
a_{t}+c_{a} a_{z}=V_{3} f b, \quad f_{t}=-V_{3} a b^{*}, \\
b_{t}-c_{b} b_{z}=-V_{3} a f^{*}+\imath R|b|^{2} b-\imath \kappa b_{t t} .
\end{gathered}
$$

Here $a, b$ and $f$ are envelopes respectively of the pump pulse, of the counter-propagating shorter pumped pulse or seed pulse, and of the resonant Langmuir wave. The subscripts $t$ and $z$ signify time and space derivatives; $c_{a}$ and $c_{b}$ are group velocities of the pump and amplified pulses; $V_{3}$ is the 3 -wave coupling constant (real for appropriately defined wave envelopes); $R$ is the coefficient of nonlinear frequency shift due to the relativistic electron nonlinearity; $\kappa=c_{b}^{\prime} / 2 c_{b}$ is the group velocity dispersion coefficient; and $c_{b}^{\prime}$ is the derivative of the amplified pulse group velocity over the frequency. The relativistic electron nonlinearity is taken into account only for the seed pulse, because the seed is ultimately amplified to intensities much higher than that of the pump. The group velocity dispersion is also taken into account only for the seed pulse, because the seed is of much shorter duration than the pump, and therefore more sensitive to the dispersion effect. 


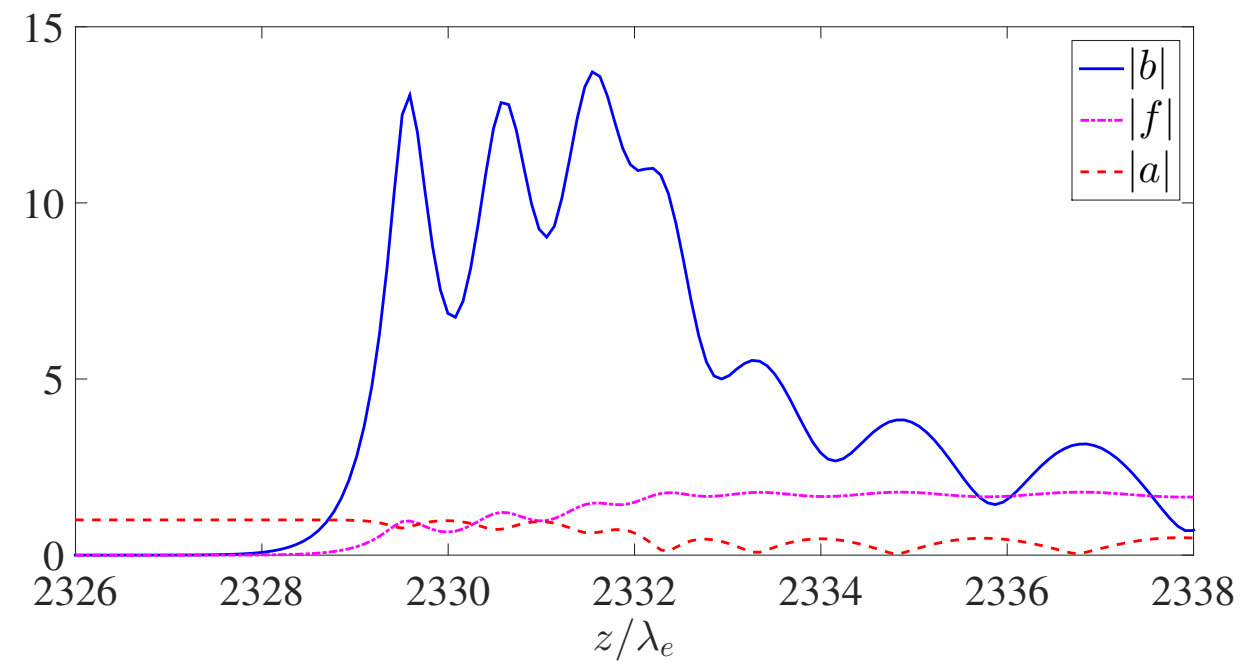

Figure 2. Envelopes of the pump (red curve), the seed (blue curve), and the Langmuir wave (magenta curve) when the seed has traversed a length of $27.5 \mathrm{~cm}$. The pump wavelength is $0.238 \mu \mathrm{m}$ and the laser pump frequency to the Langmuir wave frequency ratio is 40 . To show on the same plot, the amplified seed is scaled down by actor of 10 . Note that the third spike of the seed exceeds the first spike amplitude. The output intensity of the third spike is $0.78 \mathrm{EW} / \mathrm{cm}^{2}$ with FWHM duration of 99 fsec.

One solution to Eqs. (1-2) is the $\pi$-pulse solution, namely the solution describing complete pump depletion, obtained in the limit of a seed with a very sharp rise time, and neglecting both the group velocity dispersion and the relativistic self-focusing. The $\pi$-pulse solution is one, but not the only, solution to the three-wave coupling between two light waves and a cold plasma wave. These equations also can describe in different limits both how compression solutions can be destroyed by various deleterious effects. These equations also can describe how proper preparation of the lasers and the plasma might avoid these deleterious effects. Indeed, it is important that both the plasma and the seed be carefully prepared. ${ }^{10}$

To describe the resonant Raman amplification in the wavebreaking regime, kinetic effects must be considered, something not captured in Eqs. (1-2). To capture also kinetic effects, we employ a one-dimensional VlasovMaxwell (VM) equation solver. The VM code employed here of course recovers the solutions of the 3-wave fluid equations, Eqs. (1-2), which are valid in regimes that are far from wavebreaking, but it also captures the kinetic effects that fluid codes based on Eqs. (1-2) could not describe.

To describe the wavebreaking regime, in addition to VM codes, PIC codes can be used. Hybrid codes, which treat the laser pulses through an envelope approximation, while treating the plasma wave through PIC, do reduce the computational overhead, making the approach particularly useful for simulating multidimensional effects. ${ }^{38}$ However, in the strong wavebreaking regime, the validity of the envelope approximations might still need to be verified independently. Here, the interest is in just $1 \mathrm{D}$ effects, but in the strong wavebreaking regime. Thus, $2 \mathrm{D}$ effects can be ignored, and the 1D VM approach is computationally acceptable if not particularly fast, while capturing the key physical effects of interest here.

\section{SECONDARY SPIKE REGIME}

First, we discuss issues in how large can be the output seed pulse intensities in regimes in the most favorable regimes, namely those free of wavebreaking., and which exhibit the $\pi$-pulse solution. The current thinking has been that the maximum seed output intensity is obtained when the leading spike of the amplified seed reaches its maximum intensity. At that point, because of relativistic nonlinearities, the leading spike of the $\pi$-pulse solution, where most of the energy is captured, will then break up. Thus, the recommended strategy has been that the plasma is terminated (kept short enough) so the amplification process does not proceed for so long that this pulse is spoiled. 


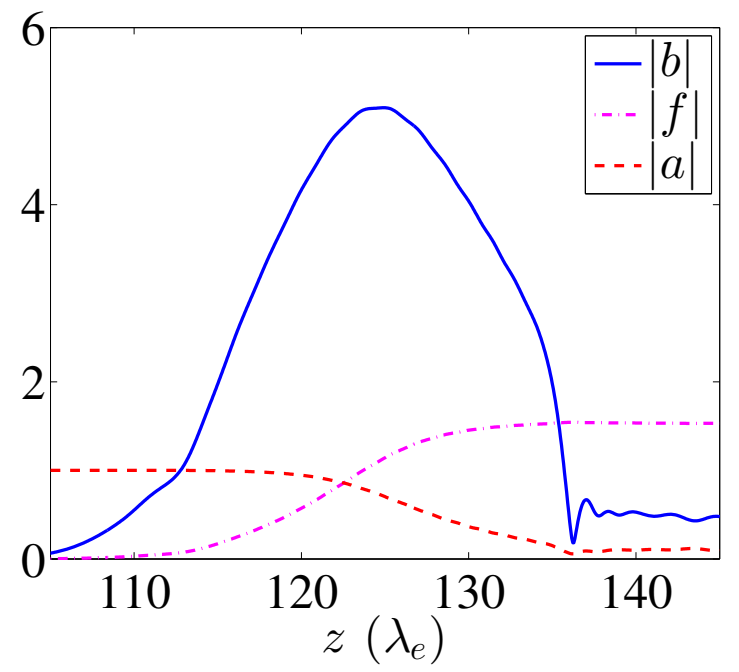

Figure 3. The envelopes of the pump (red curve), the seed (blue curve), and the Langmuir wave (magenta curve) when the seed has traversed a length of $275 \mu \mathrm{m}$. The pump wavelength is $0.351 \mu \mathrm{m}$ and the laser pump frequency to the Langmuir wave frequency ratio is 3 . When the seed dispersion becomes a dominant effect, the amplified pulse is transformed to a solitary pulse. The output intensity is $0.9 \mathrm{EW} / \mathrm{cm}^{2}$ with about duration of 52.8 fsec.

We recently found, however, that after the saturation of this leading amplified spike, the initially small secondary spikes can outgrow the leading spike and reach substantially larger intensities. ${ }^{13}$ If the plasma density is small enough so that the group velocity dispersion of the seed is negligible, after the leading spike reaches its saturation value, ${ }^{12}$ the trailing spikes can be amplified to a value which exceeds the amplitude of the leading spikes. This occurs at amplified seed pulse intensities substantially beyond the conventional limits attributed to amplification in the $\pi$-pulse regime, based on saturation of the leading spike, when the relativistic nonlinearity effects break up the pulse. ${ }^{23}$ If other deleterious processes can be avoided, however, then in fact a factor of several may then be possible in laser output intensities through the secondary spikes. To access this regime, first all deleterious effects, other than the longitudinal modulation instability, need to be controlled. The controlling techniques involve the choosing of parameter regimes, and selective detuning of the Raman resonance laser pulses and plasmas, applying appropriate chirps to the pump and seed lasers, as well as operating in a plasma density gradient. Without avoiding the deleterious effects, this regime would not be reached, whether in experiments or PIC simulations, since PIC simulations also have an inherent noise. But in avoiding the deleterious effects, the plasma length might be extended, so that the Raman amplification can result in either higher output intensity or higher output fluence.

The general case has been discussed previously. ${ }^{13}$ Here we first show an illustrative example of a very long plasma interaction. Consider an injected pump intensity of $40 \mathrm{TW} / \mathrm{cm}^{2}$ with wavelength of $0.238 \mu \mathrm{m}$ is injected into $30 \mathrm{~cm}$ long plasma. The initial counter-propagating seed has intensity of $1 \mathrm{PW} / \mathrm{cm}^{2}$ with duration of 33 fsec. The plasma density is $n_{e}=1.12 \times 10^{19} \mathrm{~cm}^{-3}$. Fig. 2 shows the envelopes of the pump (red curve), the seed (blue curve), and the Langmuir wave (magenta curve) when the seed has traversed a length of $27.5 \mathrm{~cm}$. Note how the third spike of the seed exceeds the first spike amplitude. In this case, the output intensity of the third spike is $0.78 \mathrm{EW} / \mathrm{cm}^{2}$, with full width at half maximum (FWHM) duration of $99 \mathrm{fsec}$.

As a second example, consider an injected pump intensity of $55 \mathrm{PW} / \mathrm{cm}^{2}$ with a wavelength of $0.351 \mu \mathrm{m}$, injected into a $354 \mu \mathrm{m}$ long plasma. The initial counter-propagating seed has intensity of $4 \mathrm{PW} / \mathrm{cm}^{2}$ with duration of 13.2 fsec. The plasma density is $n_{e}=8 \times 10^{20} \mathrm{~cm}^{-3}$. Fig. 3 shows the envelopes of the pump (red curve), the seed (blue curve), and the Langmuir wave (magenta curve) when the seed has traversed a length of $275 \mu \mathrm{m}$. Note that, here, when the seed dispersion becomes a dominant effect, the amplified pulse appears as a single spike. In this case the output intensity is $0.9 \mathrm{EW} / \mathrm{cm}^{2}$, with a duration of about 52.8 fsec. 

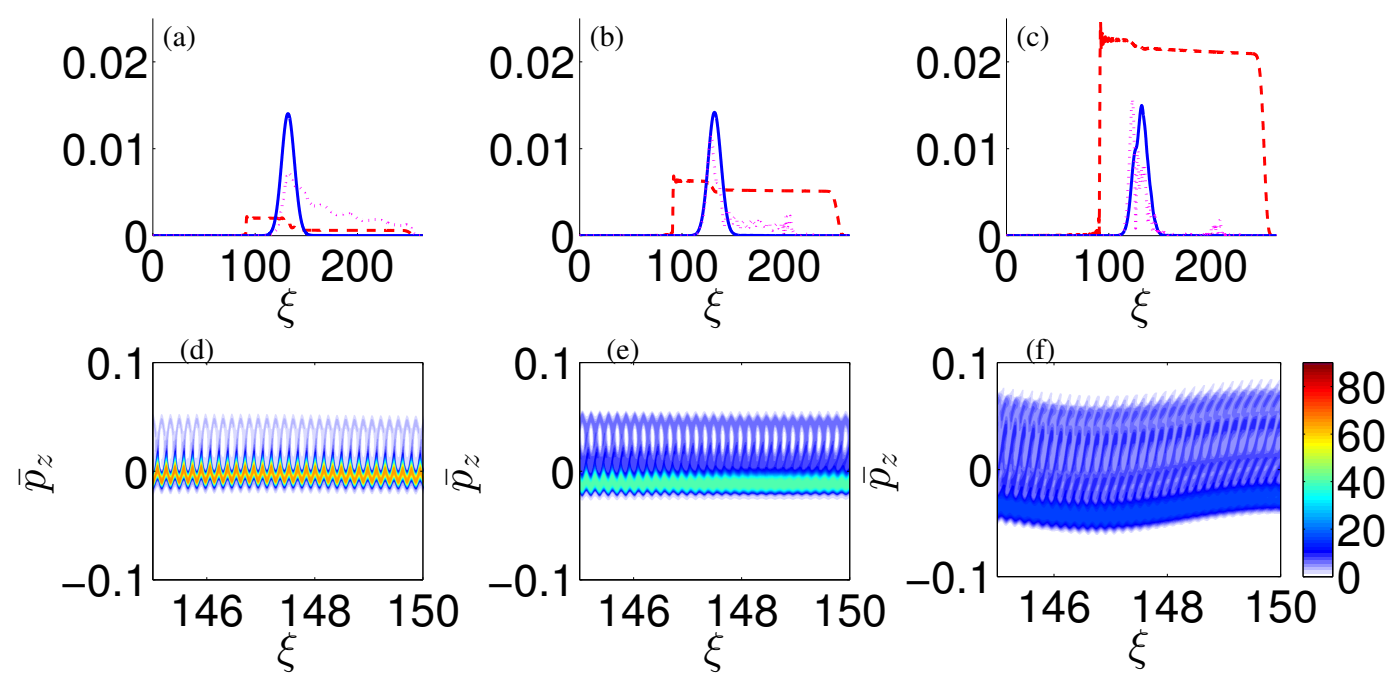

Figure 4. (a) Envelopes of the pump (dashed curve), the seed (solid curve), and the Langmuir wave (dotted curve) for pump frequency to plasma frequency ratio 20, initial electron temperature $10 \mathrm{eV}$, and pump amplitude $v_{e a} / v_{b r}=0.5$, which is below the wavebreaking threshold; (b) pump amplitude $v_{e a} / v_{b r}=1.5$, which is in the mild wavebreaking regime; (c) pump amplitude $v_{e a} / v_{b r}=5.5$, which is in the strong wavebreaking regime. (d)-(f) The corresponding electron phase space distributions in the region behind the seed pulse maximum spike.

\section{WAVEBREAKING REGIME}

Now we will consider backward Raman amplification in the Langmuir wavebreaking regime. ${ }^{14}$ As an example we consider the case where $\omega_{a} / \omega_{e}=20$. We consider an electron temperature of $10 \mathrm{eV}$, corresponding to low Landau damping. At the same time, since the Vlasov Maxwell model does not capture collisional effects, so collisional damping that might enter at low temperature will not obscure the wavebreaking effects that we wish to study.

In the regime where the initial pump amplitude is below the wavebreaking threshold $\left(v_{e a} / v_{b r}=0.5\right)$ the pump depletion is $90 \%$ (Fig. 4a). For initial pump amplitude that is a few times larger than the wavebreaking threshold $\left(v_{e a} / v_{b r}=1.5\right)$ the pump depletion is reduced to about $30 \%$ (Fig. $\left.4 \mathrm{~b}\right)$. For pump amplitude significantly larger than the wavebreaking threshold $\left(v_{e a} / v_{b r}=4.5\right)$ the pump depletion is reduced to about $10 \%$ (Fig. 4c). While in the regime below the wavebreaking (Fig. 4d) and in the mild wavebreaking regime (Fig. 4e) most of the electrons are distributed according to the initial Maxwellian distribution, in the strong wavebreaking regime, a large fraction of particles are accelerated (Fig. 4f).

In Fig. 5, we compare calculations of the pump depletion by various numerical simulations with each other and with analytical expectations. The blue curve describes pump depletion using our VM code; the green and red crosses describe PIC simulations of Ref. 35); and the magenta curve describes the analytical estimates of Ref. 4. It can be seen that there is a good agreement throughout between the VM results and the analytical estimates. In addition, most of the PIC simulations are also in reasonable agreement with the theoretical estimate. However, in the deep wavebreaking regime, where $v_{e a} / v_{b r}=5.5$, there is a large discrepancy between the PIC and the theoretical estimate. Because this deep wavebreaking regime is very interesting, it is important to understand the origin of this discrepancy. Since the VM code and the 2D PIC code might not be directly comparable, it would be worthwhile to benchmark these codes against each other. 


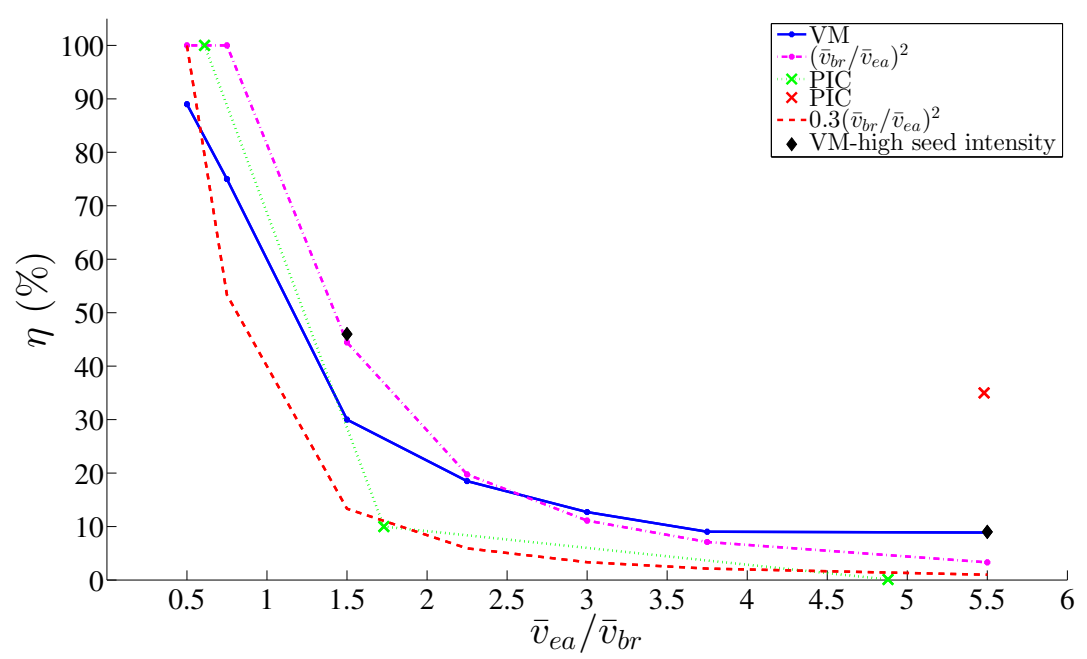

Figure 5. Pump depletion parameter as function of wavebreaking parameter. Blue curve describes our VM code; green and red crosses describe PIC simulations of Ref. 35; magenta curve describes analytical estimates of Ref. 4.

\section{SUMMARY}

Two limiting effects occur when resonant Raman compression is practiced at high intensity. The first effect addressed is the relativistic effect that causes the saturation of the seed pulse at relativistic seed pulse intensities. ${ }^{12}$ Interestingly, the saturation of the secondary spikes occurs at higher intensities than does the saturation of the leading spike, which means that for a longer plasma, greater seed pulse output intensities can be reached. ${ }^{13}$ This result requires that other deleterious effects are already mitigated, so that the limiting effect occurs from the longitudinal modulation instability. The reaching of higher output seed pulse intensities would, of course, be a considerable technological advance. The second high-intensity effect addressed is when the pump intensity is so high that wavebreaking occurs. This regime could be advantageous, so long as the pump depletion is not too diminished. We simulated these regimes using a VM code. ${ }^{14}$ In the mild wavebreaking regime, relatively high pump depletion could still be obtained, with simulations using our VM code reaching similar conclusions to those in the archival literature. In the deep wavebreaking regime, there appears to be some variance within the archival literature, with our 1D VM code suggesting considerably smaller pump depletion than that reported using simulations with a 2D PIC code. Our 1D code does, however, reproduce analytical scaling predictions. ${ }^{4}$ The reason for this apparent difference in pump depletion, in the deep wavebreaking regime, is not yet understood.

\section{REFERENCES}

[1] Strickland, D. and Mourou, G., "Compression of amplified chirped optical pulses," Opt. Commun. 56, 219 (1985).

[2] Yakovlev, I. V., "Stretchers and compressors for ultra-high power laser systems," Quantum Electronics 44, 393 (2014).

[3] Kawanaka, J., Miyanaga, N., Azechi, H., Kanabe, T., Jitsuno, T., Kondo, K., Fujimoto, Y., Morio, N., Matsuo, S., Kawakami, Y., Mizoguchi, R., Tauchi, K., Yano, M., Kudo, S., and Ogura, Y., "3.1-kJ chirpedpulse power amplification in the LFEX laser," J. Phys. Conf. Ser. 112, 032006 (2008).

[4] Malkin, V. M., Shvets, G., and Fisch, N. J., "Fast compression of laser beams to highly overcritical powers," Phys. Rev. Lett. 82, 4448 (1999).

[5] Malkin, V. M., Shvets, G., and Fisch, N. J., "Detuned raman amplification of short laser pulses in plasma," Phys. Rev. Lett. 84, 1208 (2000).

[6] Solodov, A. A., Malkin, V. M., and Fisch, N. J., "Random density inhomogeneities and focusability of the output pulses for plasma-based powerful backward Raman amplifiers," Phys. Plasmas 10, 2540 (2003). 
[7] Balakin, A. A., Fraiman, G. M., Fisch, N. J., and Malkin, V. M., "Noise suppression and enhanced focusability in plasma Raman amplifier with multi-frequency pump," Phys. Plasmas 10, 4856 (2003).

[8] Berger, R. L., Clark, D. S., Solodov, A. A., Valeo, E. J., and Fisch, N. J., "Inverse bremsstrahlung stabilization of noise in the generation of ultrashort intense pulses by backward Raman amplification," Phys. Plasmas 11, 1931 (2004).

[9] Tsidulko, Y. A., Malkin, V. M., and Fisch, N. J., "Suppression of superluminous precursors in high-power backward Raman amplifiers," Phys. Rev. Lett. 88, 235004 (2002).

[10] Malkin, V. M. and Fisch, N. J., "Key plasma parameters for resonant backward Raman amplification in plasma," Eur. Phys. J. Special Topics 223, 1157 (2014).

[11] Toroker, Z., Malkin, V. M., and Fisch, N. J., "Seed laser chirping for enhanced backward Raman amplification in plasmas," Phys. Rev. Lett. 109, 085003 (2012).

[12] Malkin, V. M., Toroker, Z., and Fisch, N. J., "Saturation of the leading spike growth in backward Raman amplifiers," Phys. Plasmas 21, 093112 (2014).

[13] Malkin, V. M., Toroker, Z., and Fisch, N. J., "Exceeding the leading spike intensity and fluence limits in backward Raman amplifiers," Phys. Rev. E, 063110 (2014).

[14] Toroker, Z., Malkin, V. M., and Fisch, N. J., "Backward Raman amplification in the Langmuir wavebreaking regime," Phys. Plasmas 21, 113110 (2014).

[15] Shvets, G., Fisch, N. J., Pukhov, A., and Meyer-ter-Vehn, J., "Supperradiant amplification of an ultrashort laser pulse in a plasma by a counterpropagating pump," Phys. Rev. Lett. 81, 4879 (1998).

[16] Andreev, A. A., Riconda, C., Tikhonchuk, V. T., and Weber, S., "Short light pulse amplification and compression by stimulated Brillouin scattering in plasmas in the strong coupling regime," Physics of Plasmas 13(5), 053110 (2006).

[17] Weber, S., Riconda, C., Lancia, L., Marques, J., Mourou, G. A., and Fuchs, J., "Amplification of ultrashort laser pulses by brillouin backscattering in plasmas," Phys. Rev. Lett. 111, 055004 (2013).

[18] Riconda, C., Weber, S., Lancia, L., Marques, J., Mourou, G. A., and Fuchs, J., "Spectral characteristics of ultra-short laser pulses in plasma amplifiers," Physics of Plasmas 20, 083115 (2013).

[19] Kruer, W. L., [The Physics of Laser Plasma Interactions], Addison-Wesley, Reading, MA (1988).

[20] Fisch, N. J. and Malkin, V. M., "Generation of ultrahigh intensity laser pulses," Phys. Plasmas 10, 2056 (2003).

[21] Clark, D. S. and Fisch, N. J., "Operating regime for a backward Raman laser amplifier in preformed plasma," Phys. Plasmas 10, 3363 (2003).

[22] Malkin, V. M. and Fisch, N. J., "Manipulating ultra-intense laser pulses in plasmas," Phys. Plasmas 12, 044507 (2005).

[23] Malkin, V. M., Toroker, Z., and Fisch, N. J., "Laser duration and intensity limits in plasma backward Raman amplifiers," Phys. Plasmas 19, 023109 (2012).

[24] Toroker, Z., Malkin, V. M., Balakin, A. A., Fraiman, G. M., and Fisch, N. J., "Geometrical constraints on plasma couplers for Raman compression," Phys. Plasmas 19, 083110 (2012).

[25] Ping, Y., Geltner, I., Fisch, N. J., Shvets, G., and Suckewer, S., "Demonstration of ultrashort laser pulse amplification in plasmas by a counterpropagating pumping beam," Phys. Rev. E 62, R4532 (2000).

[26] Ping, Y., Geltner, I., Morozov, A., Fisch, N. J., and Suckewer, S., "Raman amplification of ultrashort laser pulses in microcapillary plasmas," Phys. Rev. E 66, 046401 (2002).

[27] Ping, Y., Cheng, W., Suckewer, S., Clark, D. S., and Fisch, N. J., "Amplification of ultrashort laser pulses by a resonant Raman scheme in a gas-jet plasma," Phys. Rev. Lett. 92, 175007 (2004).

[28] Balakin, A. A., Kartashov, D. V., Kiselev, A. M., Skobelev, S. A., Stepanov, A. N., and Fraiman, G. M., "Laser pulse amplification upon Raman backscattering in plasma produced in dielectric capillaries," JETP Lett. 80, 12 (2004).

[29] Cheng, W., Avitzour, Y., Ping, Y., Suckewer, S., Fisch, N. J., Hur, M. S., and Wurtele, J. S., "Reaching the nonlinear regime of Raman amplification of ultrashort laser pulses," Phys. Rev. Lett. 94, 045003 (2005).

[30] Ren, J., Li, S., Morozov, A., Suckewer, S., Yampolsky, N. A., Malkin, V. M., and Fisch, N. J., "A compact double-pass Raman backscattering amplifier/compressor," Phys. Plasmas 15, 056702 (2008). 
[31] Vieux, G., Lyachev, A., Yang, X., Ersfeld, B., Farmer, J. P., Brunetti, E., Issac, R. C., Raj, G., Welsh, G. H., Wiggins, S. M., and Jaroszynski, D. A., "Chirped pulse Raman amplification in plasma," New J. Phys. 13, 063042 (2011).

[32] Yang, X., Vieux, G., Brunetti, E., Farmer, J. P., Ersfeld, B., Wiggins, S. M., Issac, R. C., Welsh, G. H., and Jaroszynski, D. A., "Experimental investigation of chirp pulse raman amplification in plasma," Proc. SPIE 8075, 80750G (2011).

[33] Yampolsky, N. A., Fisch, N. J., Malkin, V. M., Valeo, E. J., Lindberg, R., Wurtele, J., Ren, J., Li, S., Morozov, A., and Suckewer, S., "Demonstration of detuning and wavebreaking effects on Raman amplification efficiency in plasma," Phys. Plasmas 15, 113104 (2008).

[34] Yampolsky, N. A. and Fisch, N. J., "Limiting effects on laser compression by resonant backward Raman scattering in modern experiments," Phys. Plasmas 18, 056711 (2011).

[35] Trines, R. M. G. M., Fiuza, F., Bingham, R., Fonseca, R. A., Silva, L. O., Cairns, R. A., and Norreys, P. A., "Simulations of efficient Raman amplification into the multipetawatt regime," Nature Phys. 7, 87 (2011).

[36] Malkin, V. M., Fisch, N. J., and Wurtele, J. S., "Compression of powerful x-ray pulses to attosecond durations by stimulated Raman backscattering in plasmas," Phys. Rev. E 75, 026404 (2007).

[37] Malkin, V. M. and Fisch, N. J., "Relic crystal-lattice effects on Raman compression of powerful x-ray pulses in plasmas," Phys. Rev. Lett. 99, 205001 (2007).

[38] Farmer, J. P. and Pukhov, A., "Fast multidimensional model for the simulation of Raman amplification in plasma," Phys. Rev. E 88, 063104 (2013). 